\title{
EVALUATION OF IN VITRO SYNERGY BETWEEN AMIPICILLIN AND KANAM YCIN AGAINST STAPHYLOCOCCUS AUREUS
}

Divyashree BC, *Vishwanatha T, Spoorthi N Jain, Reena V, Aishwarya Sampath, Siddhalingeshwara KG, Venugopal N

Dept of Microbiology, Maharani’s Science College for Women, Ban galore 560001

Corresponding Author's E-mail: lvphaget@rediffmail.com

Received 04 May 2012; Review Completed 25 May 2012; Accepted 26 June 2012, Available online 15 July 2012

\begin{abstract}
ABS TRACT
The emergence of antibiotic resistance in bacteria is threatening as in case of Staphylococcus aureus and is well known for causing nosocomial infections in humans. The main objective of this study was to find ways to inhibit the growth of nosocomial infection causing S.aureus using antibiotic combinations. In this study a total of 50 isolates of S.aureus were isolated from clinical samples collected from wound, boils, abscesses, ulcers and post operative infections (POI). The isolate exhibiting multiantibiotic resistance was subjected for test of synergism. Synergism can be best achieved by checkerboard or Fractional Inhibitory Concentration (FIC). A combination of ampicillin and kanamycin were used for the checkerboard method. From the checkerbo ard, the MIC of ampicillin and kan amy cin alone were $160 \mu \mathrm{g} / \mathrm{ml}$ and $80 \mu \mathrm{g} / \mathrm{ml}$ respectively. However a combination of both antibiotics required were just $10 \mu \mathrm{g} / \mathrm{ml}$ and $20 \mu \mathrm{g} / \mathrm{ml}$ of ampicillin and kan amy cin with a FIC index of 0.562 indicating their synergistic effect. Thus from this study it can be concluded that antibiotic combination therapy is the best way to achieve synergy when the causal organism is a multi-antibiotic resistant one.
\end{abstract}

Key words: Antibiotic resistance, Staphylococcus aureus, Checker board and Synergy test.

\section{INTRODUCTION}

Among the various infections caused by bacteria, antibiotic resistant ones are of major concern because of their non-responsiveness to treatment with a single drug regimen thus resulting in therapeutic failure ${ }^{\mathbf{1}}$. The use of antibiotic combinations has been known since a long time and is often applied when several mechanis ms of action and toxicity profiles of the agents involved can be brought to halt at once ${ }^{2}$. The biocidal (bactericidal, fungicidal or virucidal) activity could be best achieved by the combination of two different antibiotics rather than the effect obtained by an individual antibiotic ${ }^{3}$. Combination of antibiotics is one such methodology and is applied only under certain circumstances. Although the combination of two or more antibiotics may not provide accurate control as observed in few cases, it can atleast delay the emergence of bacterial resistance ${ }^{4}$. Synergistic combinations are often applied in situations of resistance development and when ineffectiveness of a single antibiotic are prevalent, in treatment of life threatening infections based completely on experimental terms, prior to the identification of the causal organism, prevention of bacterial resistance development and in the treatment of infections caused by a combination of microbes (bacteria) 3. Staphylococcus spp being well known for causing nosocomial infections, it could also lead to severe clinical manifestations like pneumonia, deep abscesses, osteomyelitis, endocarditis, phlebitis, arthritis, mastitis and meningitis, thus stressing the need for combination therapy. It can also produce beta-lactamase enzyme which is capable of penicillin resistance $\mathbf{5 , 6}$. Thus, there is a need to find new ways to control evolving of drug resistant $S$. aureus infections and embark on the need for a continued search of new methods for treatment with the application of comb ination therapy ${ }^{7}$.

Among the techniques employed in the evaluation of the combination of two antimicrobials potentially exhibiting synergism, is the checkerboard or fractional inhibitory concentration (FIC) technique. FIC employs a methodology similar to that utilized for the determination of the minimum inhibitory concentration (MIC). The combination is said to have synergistic effect if there is a 4-fold reduction in the MIC of each antimic robial agent tested alone ${ }^{2}$. Kanamycins are members of the aminoglycoside family having a broad spectrum of activity against many species of bacteria. Their mechanism of action is specific to aerobic, Gram negative bacteria however it can act synergistically against some Gram positive bacteria. Kanamycin mainly targets the proteins synthesis by irreversibly binding to the 16s rRNA of 30 s ribosomal subunit. Binding of kanamycin to the decoding region of 3 end of rRNA prevents the tRNA from binding to the ribosomal A-site thus bringing about translational errors ${ }^{8}$. Ampicillin is a commonly used broad-spectrum aminopenicillin with known activity against $E$. coli and Staphylococcus aureus ${ }^{4}$. These aminopenicillins inhibit the final stage of synthesis of cross - links of peptidoglycan which occurs outside the cell and is catalyzed by a transpeptidase ${ }^{\mathbf{1}}$. In this study, the interaction between ampicillin and kanamycin is investigated using the checkerboard method. The result of the study could provide rational basis for clinical use of these combination of antibiotics against infections caused by these drug resistant organisms.

\section{METHODS}

\section{Materials:}

The culture media used in the study included Nutrient agar, Mueller-Hinton agar, Mueller-Hinton broth and Mannitol salt agar (all of them from Hi-Media). A mp icillin and Kanamycin were obtained from Hi-Media. Clinical is olates of microorganis ms were isolated from clinical samples. 


\section{Preparation of culture media:}

The culture media were prepared according to the manufacturers' specifications. Initially they were weighed, dissolved in required amount of water and then sterilized by autoclaving at $121^{\circ} \mathrm{C}$ for $15 \mathrm{~min}$.

\section{Isolation and confirmation of S.aureus:}

The clinical samples (50 samples) for the isolation of S.aureus were collected from various laboratories of Bangalore during the period October 2011 to December 2011. These isolates were initially grown on nutrient agar. The colonies on nutrient agar were identified based on colony characteristics and Gram staining. Further characterization was carried out by streaking the inoculum on selective media i.e., Mannitol salt agar. The organism was confirmed by performing biochemical tests, most important of them are catalase and coagulase tests both of which gave positive results. The isolated and confirmed isolates were subcultured weekly on fresh nutrient agar slants and stored at $4^{\circ} \mathrm{C}^{3} .24 \mathrm{hr}$ fresh culture was obtained prior to each study.

\section{Antibi otic susceptibility test:}

Antimicrobial susceptibility was determined by KirbyBauer disc diffusion technique. Antibiotic discs used in this investigation are standardized discs: Kanamycin (30 mcg/disc), Chloramphenicol (30 mcg/disc), A mpicillin (30mcg/disc), Vanco mycin (30mcg/disc), Erythromycin (15mcg/disc) and Penicillin (10units/disc). Actively growing $16 \mathrm{hr}$ old culture was surface spread using sterile cotton swabs onto the Mueller-Hinton agar surface. The plates were kept aside for absorption for $15 \mathrm{~min}$. The antibiotic discs were then placed onto the agar surface and the plates were incubated at $37^{\circ} \mathrm{C}$ for $16-18 \mathrm{hr}$. The plates were examined for the zone of inhibition around the antibiotic discs that would indicate the sensitivity of the organis $\mathrm{m}^{1}$.

\section{Preparation of antibi otic stock solution:}

Standard powder forms of Ampicillin and Kanamycin were stored at 2 to $8^{\circ} \mathrm{C}$ until use. The stock solution of Ampicillin and Kanamycin was prepared by weighing and subsequently dissolving appropriate quantities of the antibiotic in $50 \mathrm{ml}$ of Mueller- Hinton broth to obtain a concentration of $1000 \mu \mathrm{g} / \mathrm{ml}$ of each antibiotic.

\section{Preparation of inoculum:}

The inoculum was obtained by transferring a small volu me of stored culture to fresh media which was incubated overnight containing actively dividing cells. This was used for synergism test.

\section{Test for Synergism:}

Serial two fold dilutions of each antibiotic stock to at least double the MIC were prepared. The first antibiotic of the combination was serially diluted along the ordinate while the second antibiotic was diluted along the abscissa? Varying concentrations of 2.5, 5.0, 10, 20, 40, 80, 160 and $250 \mu \mathrm{g} / \mathrm{ml}$ of each antibiotic was used for combination. A total of $2000 \mu 1$ of Mueller -Hinton broth was distributed into each $2.5 \mathrm{ml}$ screw cap tubes which also contained $100 \mu \mathrm{l}$ of the bacterial culture. The tubes were incubated at $37^{\circ} \mathrm{C}$ for $24 \mathrm{hr}$. The resulting checker board contains a combination of two antibiotics, with the tubes that contain the highest concentration of each antibiotic at opposite corners ${ }^{9}$.

According to the NCCLS guidelines for broth microdilution, the MIC was defined as the lowest concentration of antibiotic that completely inhibited the growth of the organism as observed detected with the naked eye. Synergy is more likely to be expressed when the ratio of the concentration of each antibiotic to the MIC of that antibiotic was same for all components of the mixture. Interaction was assessed algebraically by determining the fractional inhibitory concentration (FIC) indices. The FIC (Fractional Inhibitory Concentration) were calculated as follows: $\mathrm{FIC}=$ FIC A+ FIC B, where FIC $\mathrm{A}$ is the MIC of antibiotics in combination/ MIC OF antibiotic A alone and FIC B is the MIC of antibiotics in combination/ MIC OF antibiotic B alone.

\section{RES ULTS}

The clinical samples were collected for the study from different laboratories ${ }^{\mathbf{6}, \mathbf{1 0}}$. A maximum of $36 \%$ clinical samples were obtained from wound and abbesses and post operative infections (POI) showed $21 \%$ followed by ulcer $13 \%$ and boils $9 \%$. Results show incidence of $S$. aureus infections (Fig 1).

The disk diffusion test plates were examined for zone of inhibition around the antibiotic disks. Thus disk diffusion results indicate the multi drug resistance pattern of Staphylococcus aureus. The development of resistance by $S$. aureus is an ever increasing problem. Hence a combination of antibiotics using checker board method gave better control against S.aureus ${ }^{11,12}$.

In the Checkerboard technique, the interaction between combination of ampicillin and kanamycin against Staphylococcus aureus were predominantly synergistic with FIC index of 0.5625 , although there were few variations. Thus no growth or turbidity clearly illustrated the extensive activity of aminoglycoside which was enforced by the second drug, ampicillin resulting in an antibacterial effect (Fig.2). FIC-index values less than 1 were considered as synergy and the degree of synergy increases as the value tends towards zero. FIC-index value greater than one but less than two indicates indifference while FIC value greater than two represents antagonism.

\section{DISCUSS ION}

Goals of combination therapy are to obtain broad spectrum coverage, enhance antimicrobial activity through synergistic interaction and minimize resistance development for most infections. A combination therapy may be needed for treatment of mixed infections, when not all organisms are susceptible to the same antibiotic; prevention or delay of development of bacterial resistance to an antibiotic; ability to use non-toxic amounts of two antibiotics when toxic doses of a single antibiotic would be required and when combination therapy may be more effective against a single organism than the use of single antimicrobial agent.

In this study the FIC-index for combination of ampicillin and kanamycin against S.aureus was found to be 0.5625 indicating the synergistic effect of the combination, the FIC value being in agreement with the report of Nworu et al report (2006) the combination being ampicillin and ciprofloxacin. This report is also in contradiction with that 
of Ibezim et al (2006) using ampicillin and cotrimoxazole combination. This is because the combination used could vary depending upon the strain of S.aureus, the environment in which they exist and several other conditions. The combination of two antibiotics exhibited significant synergism, the mechanism involving the penetration of ampicillin into the peptidoglycan layer leading to cross-links and inhibition of cell wall synthesis and there by increases the permeability of the bacterium to kanamycin that binds to the 30 s ribosome inhibiting the protein synthesis ${ }^{13,14}$. On the other hand the organism exhibited a higher MIC for one of the antibiotic alone and this is in support with the report of Sato et al (2004) ${ }^{15,16}$. It is hoped that these approaches, if well standardized and adopted, will not only provide useful alternatives to preexisting time-kill and checkerboard titration method, but will also circumvent problems and methodological limitations inherent in their use ${ }^{17,18}$

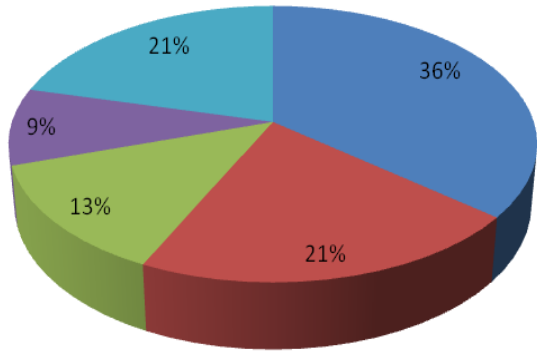

Wounds

- Abscess

Ulcers

noils

$\square \mathrm{POI}$

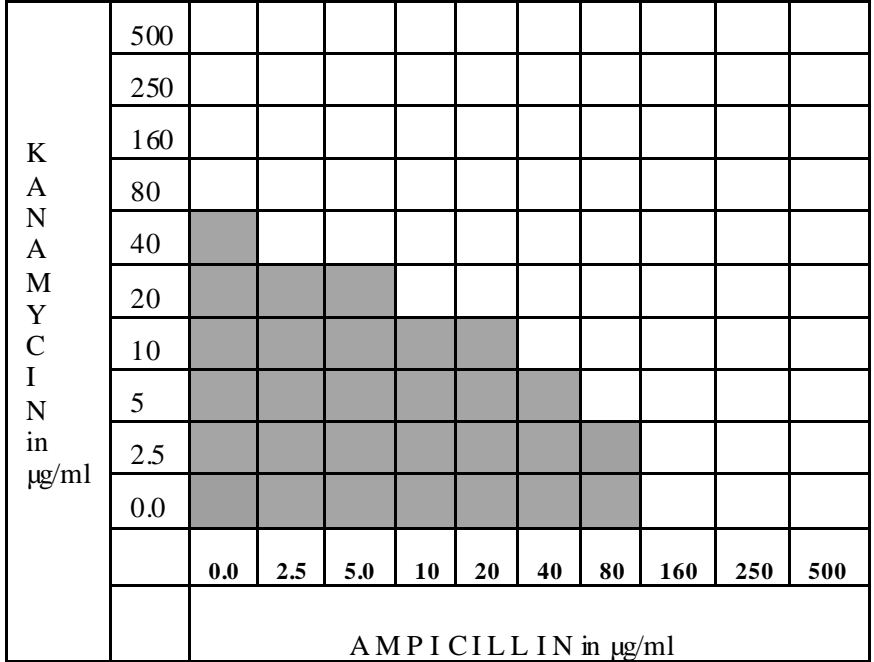

Figure 2: The synergistic effect of the two antibiotics against $S$. aureus

\section{CONCLUSION}

Staphylococcus aureus, the 'king of nosocomial infections' is of major concern all over the globe because of the multiantibiotic resistance pattern exhibited by the organism. Although researchers are finding newer antibiotics for the control of 'the nosocomial king', its multi-antibiotic resistance pattern still exists and hence synergistic effect of known antibiotics is the best tool that could be employed to control nosocomial infections caused by $S$. aureus.
Figure 1: Incidence of infection by S.aureus in clinical samples

\section{REFERENCES}

1. Ibezim EC, Esimone CO, Okorie O, Onyishi, IV, Nnamani PO, Brown, SA, Obodo CE, A study of the in vitro interaction of cotrimoxazole and ampicillin using the checkerboard method. African Journal of Biotechnology. 2006, 5:1284-1288.

2. de Souza, D. Mehta and R. W. Leavitt. Bactericidal activity of combinations of Silver-Water Dispersion ${ }^{\mathrm{TM}}$ with 19 antibiotics against seven microbial strains. Current Science. 2006, 91:926-929.

3. Lorian V, Williams and Wilkins, Baltimore. Antibiotics in Laborat ory Medicine: Laborat ory methods used to assess the activity of antimicrobial combinations. 1991, 3: 434-444.

4. C S Nworu, C O Esimone. Comparitive evaluation of three in vitro techniques in the interaction of ampicillin and ciprofloxacin against Staphylococcus aureus and E.coli. Tropical Journal of Pharmaceutical Research. 2006, 5:605-611.

5. Rybak JM and McGrath JB. Combination antimicrobial therapy for bacterial infections. Guidelines for the Clinician, Drugs, 1996, 52: $390-402$.

6. Rahima Begum, Syeda Tasneem Towhid, Mohammad Moniruzzaman, Zakaria Mia and Mohammad Ariful Islam. Study of Staphylococcus aureus from clinical samples in Savar, Bangladesh. Res. J. of. Microbiology. 2011, 6: 884-890.

7. Ce'dric Jacqueline, Dominique Navas, Eric Batard, Anne-Franc oise Miegeville, Virginie Le Mabecque, Marie-France Kergueris, Denis Bugnon, Gilles Potel, and Jocelyne Caillon. In vitro and In vivo synergistic activities of linezolid combined with subinhibitory concentrations of imipenem against methicillin-resistant Staphylococcus aureus, Antimicrobial agents and chemotherapy. 2005, 49: 45-51.

8. Reza Faraji, Ashkan Parsa, Bahar Torabi, And Todd Withrow. Effects of kanamycin on the macromolecular composition of kanamycin sensitive Escherichia coli DH5 $\alpha$ strain, Journal of experiment al microbiology and immunology. 2005, 9:31-38.

9. Gani Orhan, Aysen Bayram, Yasemin Zer and Iclal Balci. Synergy tests by E-test and checkerboard methods of antimicrobial combinations against Brucella melitensis. Journal of Clinical Microbiology. 2004, 43:140-143.

10. Balasundaram. A, P. Rathna kumara, George John and B.N. Selvakumar. Antimicrobial activity of leaf extracts against MRSA from human urinary tract pathogens. Res.J. of. Microbiology. 2011, 7: 625-631.

11. Binh An Diep, Henry F Chambers, Christoper J Graber et al Emergence of multidrug resistant community associated methicillin reisitant S.aureus clone USA 300 in men who have sex with men. Annals of internal medicine. 2008, 148: 249-257.

12. Vishwanatha $T$ and Dyanand A. Prevalence of methicillin resistant Staphylococus aureus in gulbarga, karnataka. J Microb. World. 2009, 11: 179-183.

13. Sato , Y., H. Shibata, T.Arai, A. Yamamoto, Y. Okimura, N. Arakaki and Higuti. Variation in synergistic activity by flavonone and its related compounds on the increased susceptibility of various strains of methicillin-resistant Staphylococcus aureus to beta-lactum antibiotics. Int.J. Antimicob. Agents. 2004, 24: 226-233.

14. O.O. Olajuyigbe. Synergistic influence of tetracycline on the antibacterial activities of amoxicillin against resistant bacteria, Journal of pharmacy and allied sciences. 2012, 2:12-20.

15. H.A. Saeed and W.B. Ahmed. Invitro activity of some antimicrobial agents against Staphylococcus aureus and methicillin-resistant Staphylococcus aureus in Khartoum, Sudan. Res. J. of. Microbiology. 2009, 4:366-369.

16. Cheesbrough.M. Medical Laboratory Manual for Tropical Countries. Oxford: London. 1984, Vol. II 